Environnement urbain

Urban Environment

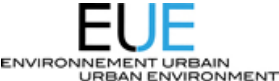

\title{
Des environnements urbains sous influence : regards croisés sur les pratiques autour du lieu de travail
}

\section{Leslie Belton-Chevallier et Benjamin Pradel}

Volume 5, 2011

URI : https://id.erudit.org/iderudit/1007606ar

DOI : https://doi.org/10.7202/1007606ar

Aller au sommaire du numéro

Éditeur(s)

Réseau Villes Régions Monde

ISSN

1916-4645 (numérique)

Découvrir la revue

Citer cet article

Belton-Chevallier, L. \& Pradel, B. (2011). Des environnements urbains sous

influence : regards croisés sur les pratiques autour du lieu de travail.

Environnement urbain / Urban Environment, 5, c22-c36.

https://doi.org/10.7202/1007606ar

\section{Résumé de l'article}

Travailler « en ville » ou à proximité de nombreuses aménités urbaines, permanentes ou temporaires, rend possible la réalisation de nombreuses activités non professionnelles à proximité de son lieu de travail. À travers deux enquêtes sociologiques complémentaires dans le quartier d'affaires de La Défense et dans les arrondissements centraux de Paris qui ont accueilli Paris-Plages, cet article propose d'examiner des pratiques socio-spatiales des travailleurs autour de leur adresse professionnelle. Les résultats qualitatifs et quantitatifs de ces enquêtes montrent que le travail reste un cadre primaire fort, à même d'influencer et de limiter le périmètre d'activités des individus à sa proximité immédiate. 


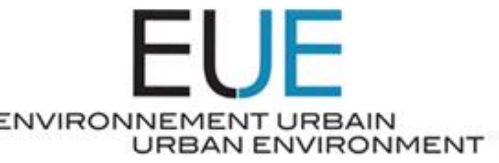

\title{
DES ENVIRONNEMENTS URBAINS SOUS INFLUENCE : REGARDS CROISES SUR LES PRATIQUES AUTOUR DU LIEU DE TRAVAIL
}

\author{
Leslie BELTON-CHEVALLIER \\ Benjamin PRADEL
}

\begin{abstract}
I RÉSUMÉ
Travailler « en ville » ou à proximité de nombreuses aménités urbaines, permanentes ou temporaires, rend possible la réalisation de nombreuses activités non professionnelles à proximité de son lieu de travail. À travers deux enquêtes sociologiques complémentaires dans le quartier d'affaires de La Défense et dans les arrondissements centraux de Paris qui ont accueilli Paris-Plages, cet article propose d'examiner des pratiques socio-spatiales des travailleurs autour de leur adresse professionnelle. Les résultats qualitatifs et quantitatifs de ces enquêtes montrent que le travail reste un cadre primaire fort, à même d'influencer et de limiter le périmètre d'activités des individus à sa proximité immédiate.
\end{abstract}

MOTS-CLÉS - Lieu de travail, pratiques, routines, cadre primaire, modalisation

\begin{abstract}
I ABSTRACT
Individuals have the opportunity to attend to a number of non-work related (or private) activities when they work "in town", or near a diverse range of urban amenities. Drawing on two joint sociological surveys conducted in the La Défense business area and in the central districts of Paris where the Paris-Plages (Paris Beaches) event takes place every year in the summer, this paper explores spatial practices of workers in the vicinity of their workplaces. The surveys yielded qualitative and quantitative results that enable us to show how work constitutes a primary framework that influences and marks the boundary close to their workplace where individuals attend to their activities.
\end{abstract}

KEYWORDS - Workplace, practices, routines, primary framework, modelling

I Coordonnées des auteurs : Leslie Belton-Chevallier, Chargée de Recherche, DEST - IFSTTAR, leslie.belton-chevallier@ifsttar.fr; Benjamin Pradel, Chargé de Recherche, LVMT École des Ponts ParisTech, benjamin.pradel@enpc.fr 


\section{INTRODUCTION}

On associe à la modernité actuelle un affaiblissement des routines individuelles. Reflets de l'action traditionnelle, ces dernières cadrent mal avec l'idéal de fluidité et de mouvement permanent de notre époque (Bauman, 2007). Pour autant, plusieurs auteurs (de Coninck, 2010 ; Delahaye et Guillot, 2010) montrent qu'il ne faut pas conclure trop vite à la disparition des routines. Derrière l'idée de routines, se trouve celle d'habitudes, d'activités répétées et fortement intériorisées par les individus. Un des niveaux dans lequel elles s'inscrivent est celui même de la vie de tous les jours (Javeau, 2006). Les individus les utilisent au quotidien en les construisant de façon réflexive (Ascher, 2004). Peu importe l'origine de ces habitudes structurantes, leur simple existence est le témoignage d'une résistance, de la nécessité de relativiser les impacts supposés de la modernité actuelle. En effet, cette dernière s'inscrit résolument dans le présent et dans l'aléatoire, symboles de liberté, refusant toute planification ou régularité : «L'homme présent » (Laïdi, 2002) valorise un «temps instantané » (Urry, 2005) en s'adaptant continuellement à des impératifs soudains (Jauréguiberry, 2003). L'idéal du temps immédiat est opposé alors au concept de routine. Avec l'instauration d'un nouveau rapport au temps, la modernité actuelle semble également s'affranchir de l'idée même de distance (Cairncross, 200I). Les activités sociales étant de moins en moins ancrées spatialement en vertu d'une mobilité débridée, la dimension spatiale semble de plus en plus éludée dans les analyses sociologiques. Ainsi, perte de sens du spatial et disparition des routines apparaissent fortement liées en vertu du culte moderne du temps immédiat.

Ces transformations rejaillissent sur les modalités d'inscription spatiotemporelle du travail en tant qu'activité située. Le travail est un contexte d'action dès lors qu'il est interprété sous l'angle de la production d'un «cadre de l'expérience » (Goffman, 1991) et analysé à travers l'influence que ce cadre possède sur les pratiques sociales qui lui sont rattachées. Aujourd'hui, le travail constituerait de moins en moins un «cadre primaire structuré » (Goffman, 199I) donnant un sens spécifiques aux mobilités spatiales, aux agendas personnels et aux interactions des individus en situation professionnelle. « L'espace de proximité » (Guérin-Pace, 2003) autour de l'adresse professionnelle est abordé en termes de cadre primaire du travail analysé dans ses dimensions spatiales et temporelles, en tant qu'il se superpose à l'urbain comme espace-temps et définit un périmètre constitutif des cartes mentales de la ville. L'adresse professionnelle colore un territoire à sa proximité, quotidien et connu, où se déploient des pratiques sociales rattachées au cadre du travail. Or, dans la perspective d'une modernité débridant les rapports que l'individu entretient avec l'espace-temps, ce cadre serait de moins en moins capable « dans une situation donnée d'accorder du sens à tel ou tel de ses aspects, lequel autrement serait dépourvu de signification » (Goffman, 1991, p.30). De plus, si la force de ce cadre primaire est mesurée par sa capacité à influencer les actions individuelles, l'évolution des modalités du travail interroge son emprise sur les pratiques spatiales quotidiennes. Le travail se diluerait, serait moins attaché à un lieu et à une durée délimités. Dans le contexte de la mondialisation, l'entreprise n'est plus un lieu unifié mais plutôt une multitude de lieux. L'éclatement du périmètre des entreprises renvoie à celui de leurs clients et leurs fournisseurs. Dans le but d'entretenir des relations avec ses partenaires, une firme a aussi recours à des employés de plus en plus mobiles. Ce regain de mobilité professionnelle physique se traduit par des lieux de travail de plus en plus variables et temporaires (Crague, 2004). Avec la distance, le cadre du travail serait moins apte à réguler les comportements. II le serait d'autant moins qu'il occupe une part décroissante dans la vie des individus. La diminution tendancielle du temps de travail libère d'un temps contraint et routinier au profit des loisirs, du temps libre comme lieu de création autonome (Friedmann, cité par Javeau, 2006, p.230). Enfin, les technologies de l'information et de la communication, tels Internet ou le téléphone portable, permettraient au travailleur de s'extraire de son cadre professionnel tout en étant physiquement présent (Cette, 2004). Faire ses courses en ligne ou avoir des communications personnelles sont des activités qui peuvent être effectuées au cœur même du travail. Ces exemples font partie d'un ensemble d'évolutions récentes qui renforcent la thèse de la dissolution du travail, de ses temps et de ses lieux. Au final, le travail perdrait peu à peu son rôle structurant sur les comportements quotidiens (Viard, 2002).

Toutes ces évolutions et leurs impacts sont à relativiser. Des travaux comme ceux d'Aguiléra et al. (2010) montrent la tendance à la spécialisation des journées des actifs, entre journées de travail où ont uniquement lieu des déplacements liés au travail d'un côté et journées hors travail où ont lieu la plupart des déplacements non liés au travail. L'objectif est ici de voir comment le travail continue de structurer les comportements individuels à l'échelle de l'espace urbain. II s'agira de montrer la permanence de contraintes liées au travail et qui pèsent sur les mobilités spatiales des actifs occupés, malgré l'électivité et la liberté supposées de la modernité 
actuelle. Dans un premier temps et à partir d'une enquête qualitative, nous démontrerons la force qui relie le travailleur à son adresse professionnelle en analysant les pratiques spatiales routinières à sa proximité. Dans un second temps et à partir d'une enquête quantitative, nous testerons la résistance de cette force en évaluant les effets, sur ces mêmes pratiques, d'un aménagement ludico-festif installé à proximité du lieu de travail.

\section{MATÉRIAUX ET MÉTHODOLOGIES}

L'enquête qualitative s'est déroulée dans le quartier d'affaires de La Défense (92) en 2006. À travers 208 entretiens courts $(20$ minutes en moyenne) effectués sur place auprès de travailleurs', elle avait pour ambition de comprendre les usages du site en périphérie du travail. Au total, 100 femmes et 108 hommes ont été interrogés. Par ailleurs, l'échantillon comporte 24 cadres et 184 non cadres (professions intermédiaires principalement). Cette faible représentativité des cadres tiendrait au fait qu'ils « sortent » moins de leur lieu de travail pendant les pauses que les autres catégories et au fait que leur taux de refus soit plus important, prétextant un manque de temps. L'enquête quantitative a eu lieu sur l'opération Paris Plages en 2007, aménagement ludique et périodique des berges de la Seine et du Bassin de la Villette initié en 2002. Elle est constituée de 1509 questionnaires passés sur place. Parmi les personnes interrogées figurent 188 individus ayant un emploi et travaillant effectivement le jour de l'enquête, dont 104 femmes et 84 hommes répartis en 89 employés, 43 cadres, 32 professions intermédiaire, 20 artisans ou commerçants et seulement 4 ouvriers. Les autres catégories (inactifs, actifs inoccupés et actifs occupés ne travaillant pas le jour de l'enquête) permettent, par comparaison, de mieux saisir les comportements spatiaux des premiers.

Le dialogue entre ces deux enquêtes nous a paru fécond pour répondre à la problématique exposée plus haut. Elles s'inscrivent toutes deux dans des périmètres spatiaux clairement délimités qui se caractérisent par leur insertion dans un environnement où se concentrent de nombreuses entreprises, bureaux et commerces: quartier d'affaire sur un plateau piéton minéral pour l'une, espaces patrimoniaux en centre-ville en bord de l'eau (la Seine

\footnotetext{
${ }^{1}$ Sont considérées comme travailleurs, toutes les personnes interrogées qui étaient actives, occupées et qui travaillaient le jour de l'enquête. Par ailleurs, 56 visiteurs, c'est-à-dire des personnes qui ne travaillent pas sur le site, ont également été interviewés. Leurs réponses n'ont pas été exploitées dans le cadre du présent article.
}

et le Canal de l'Ourcq) pour l'autre. Cependant, ParisPlages, en tant qu'espace ludique, se présente a priori comme un contre-exemple de La Défense, espace dédié au travail. Pour les concepteurs de Paris-Plages, un des objectifs était de produire un lieu en rupture avec la ville du quotidien en faisant en sorte « et c'est l'intérêt de l'éphémère, que les gens croient en un espace et en très peu de temps $\|^{2}$ et en fournissant la possibilité de vivre « une échappée belle, une journée où l'emploi du temps n'est plus réglé à l'avance ». Finalement, les deux enquêtes menées convergent dans l'analyse de l'emprise du travail sur les routines spatiales autour de l'adresse professionnelle. Si La Défense est étudié comme un cas emblématique de cette emprise, Paris-Plages introduit une forme de transformation du cadre du travail qui est censée mettre à mal cette dernière. Pourtant, les deux approches se renforcent mutuellement pour démontrer qu'il n'en est rien. Trop souvent séparées, les méthodes quantitatives et qualitatives se rejoignent et s'enrichissent l'une l'autre dans cet article pour proposer une approche socio-spatiale des pratiques urbaines dans le cadre du travail.

\section{LES ROUTINES SPATIALES DE LA DÉFENSE : L'OMNIPRÉSENCE DU TRAVAIL}

L'étude menée à La Défense a mis en évidence que le travail était un puissant cadre organisateur des pratiques spatiotemporelles des individus à son voisinage. Entre attraction et répulsion, les instants périphériques que constituent les pauses et la fin de journée s'organisent selon des routines qui prennent appui sur le cadre du travail autant qu'elles sont contraintes par celui-ci.

\section{I Espace, temps de pause et force d'attraction du travail}

L'examen des pratiques habituelles du site de La Défense rend compte de l'exercice de routines d'utilisation du lieu essentiellement auprès d'employés, de professions intermédiaires et de cadres. Les routines de ces derniers, au voisinage immédiat de leurs temps de travail - c'est-à-dire pendant la pause et en repartant du site - rendent compte de la force structurante du travail à sa proximité. En débordant régulièrement de ses cadres spatiotemporels officiels (horaires et lieux), le travail reste prégnant sur les actions individuelles. Dans l'ensemble, de nombreuses activités peuvent être effectuées à La Défense: ce

2 Entretien avec J.-C. Choblet, scénographe de Paris Plages, réalisé le 23 novembre 2006. 
quartier d'affaires abrite des bureaux mais également des logements, un centre commercial, des cinémas, des restaurants, des salles de sports, des espaces verts, un grand nombre de services à la personne (banques, poste, cabinets médicaux, etc.). Cependant, au quotidien, l'éventail de ces aménités n'est pas entièrement utilisé. Les travailleurs interrogés évoquent le plus souvent des pratiques récurrentes et peu diversifiées. Manger, lire, écouter la radio, fumer une cigarette, flâner ou discuter lorsqu'ils sont en groupe sont les activités les plus fréquemment réalisées en périphérie du travail. Faire du lèchevitrine, aller déjeuner avec des relations privées extérieures, aller au cinéma sont des activités plus singulières et rares. Elles ne sont pas évoquées spontanément dans les discours et lorsqu'elles le sont, l'adverbe « occasionnellement » ou l'expression « ça peut arriver $»$ leur sont constamment associés. Elles n'entrent pas dans les routines d'utilisation du site, ces dernières restant contraintes par le travail. En dehors de leur bureau, les travailleurs se contentent d'activités qui peuvent être facilement interrompues et qui permettent un retour rapide au travail. Ce dernier n'est jamais perdu de vue par les contraintes horaires qu'il impose, mais également par le cadre architectural l'environnant. Les routines d'utilisation du lieu s'inscrivent dans le maintien d'une relative proximité spatiale entre l'individu et son bureau lors de la pause :

Je préfêre rester ici, à proximité de mon bureau. II y a des gens, je peux les regarder passer et puis c'est calme.

(Femme, 32 ans, gestionnaire)

Ce ne sont aucunement des moments de détente. Je suis toujours sur mon lieu de travail et je pense aux obligations qui m'attendent. En plus, je ne m'éloigne pas trop de la tour. Si je me balade plus loin, je ne reviens pas ensuite.

(Homme, 25 ans, comptable).

Le cadre du travail limite les possibilités d'éloignement autour de l'adresse professionnelle et restreint le champ des services et activités accessibles. Sortir du périmètre du travail s'avère ainsi difficile, même si ce dernier diffère d'un individu à l'autre. Pour certains, il est très limité et une sortie pendant la pause déjeuner ne s'avère pas automatique, ni même possible. Pour ceux qui sortent, prendre leur pause en dehors des murs de l'entreprise est un acte de coupure symboliquement fort, dont la portée est variable :

La pause déjeuner est un moment de détente, où on peut choisir d'occuper son temps comme on le souhaite. Le site s'y prête car il permet l'anonymat.

(Homme, 53 ans, consultant)
C'est difficile de considérer les pauses comme un moment de détente. Le site est beaucoup trop bétonné pour cela. On ne peut pas flâner, il y a trop de monde et pas assez de verdure. Ça reste un lieu de travail, rien de plus.

(Homme, 40 ans, agent de service au CNIT)

En examinant les différences de comportement des catégories socioprofessionnelles, les cadres apparaissent moins contraints que les autres par leur environnement immédiat. Sur un plan spatial, être cadre suppose une plus grande liberté de mouvement, une plus grande mobilité. Cette liberté contribue à relativiser la force d'attraction d'un lieu spécifique par rapport à d'autres. Outre la catégorie, le statut du travailleur importe également. Les travailleurs précaires (CDD, stagiaires, intérimaires) restent à proximité du travail. Sa force de rappel est forte dans la mesure où de tels statuts sont souvent assortis de contraintes horaires. Temporaires sur le lieu, ils n'ont pas le temps et la nécessité d'apprendre à découvrir le site. Enfin la durée de la pause joue un rôle important. Plus cette dernière est courte, moins il est possible de s'abstraire de son environnement de travail immédiat.

\subsection{L'emprise du travail sur les interactions sociales}

Les routines d'utilisation de La Défense par ses travailleurs s'avèrent orientées vers le travail au vu de la pauvreté des activités réalisées et du maintien d'une proximité spatiale avec ce dernier. Cette force d'attraction du travail se matérialise également d'un point de vue social. Les us des travailleurs laissent peu de place à l'expression de liens sociaux non professionnels. La force d'attraction du cadre professionnel est matérialisée par la faible propension de liens privés à se manifester. Les rencontres en face-à-face avec des membres de sa famille ou des amis sont rares car elles supposent une forte proximité spatiale (travailler à La Défense ou à côté) ainsi que des pauses relativement longues. Dans le premier cas, chacun reste à proximité de son emploi et la force de rappel écourte la rencontre. Dans le second cas, l'extension de la durée de pause s'avère limitée par les contraintes horaires du poste occupé. Cette faiblesse des interactions privées s'explique surtout par une inadéquation du lieu avec le lien : les liens privés sont entretenus en dehors de La Défense.

Mais les liens privés peuvent s'exprimer autrement, notamment via le téléphone portable. Là encore, les pratiques varient d'un individu à l'autre. Si certains utilisent de façon systématique leur téléphone pour garder contact avec leurs proches, d'autres ne l'utilisent jamais : 
Tous les jours j'ai mon fiancé au téléphone. (Femme, 27 ans, assistante)

Je n'ai pas le temps de manger alors je ne vais pas téléphoner.

(Homme, 39 ans, responsable de projet)

J'utilise rarement mon téléphone portable sauf quand mes enfants m'appellent en cas de problème.

(Homme, 52 ans, ingénieur en électronique)

Une différence de catégorie socioprofessionnelle s'observe. Au cadre occupé par son travail en permanence s'oppose la figure de l'employé moins sollicité. Si le dernier profite de sa pause pour rétablir un lien privé, le premier cherche à se couper de tout lien ou sera contraint par des liens professionnels. Le rétablissement du lien privé joue quand les personnes interrogées sont des travailleurs isolés, qui n'ont pas les mêmes horaires que leurs collègues ou qui ont un statut précaire et sont donc peu intégrés socialement. En règle générale, l'usage du téléphone portable est limité à des conversations brèves dans le cadre d'une "sociabilité connectée » (Licoppe, 2002). II permet aussi de répondre à une urgence. Ce mode de communication connecté se traduit différemment selon l'étape du cycle de vie. Ainsi les personnes jeunes et célibataires communiquent avec leurs amis ou relations amoureuses alors que les personnes plus âgées ou/et ayant des enfants, notamment en bas âge, en profitent pour contacter leur cercle familial proche.

L'absence de liens privés dans les routines d'occupation de La Défense marque la puissance du cadre du travail qui empêche la réalisation de liens hors contexte. Pour autant, les individus ne sont pas toujours seuls, comme en témoigne la présence de leurs collègues. Ceux-ci constituent la dimension sociale du cadre professionnel. S'ils peuvent être qualifiés de quasi privés car quasi amicaux, beaucoup de personnes soulignent la difficulté à opérer une scission avec le travail en leur présence :

Ce n'est pas vraiment de la détente pure car je pense encore au travail, les discussions tournent toujours autours de ça.

(Femme, 50 ans, informaticienne)

Le hic quand on croise quelqu'un qu'on connait, c'est qu'on parle boulot alors qu'on n'a pas forcément toujours envie. Ce n'est pas facile de couper dans ces conditions.

(Femme, 25 ans, secrétaire)
Sortir avec ses collègues revient donc à rester connecté avec le travail et renforce sa force de rappel à son voisinage immédiat. Même si ces relations peuvent être considérées comme quasi privées, elles restent attachées au cadre dont elles sont issues. S'en absoudre suppose de mettre en place des normes claires et explicites ou d'être en petits groupes avec des collègues réellement privilégiés. Les groupes qui s'établissent alors se fondent sur d'autres liens que ceux du travail (Hatzfeld, 2002).

Le cadre du travail dépasse les limites physiques de l'entreprise et se répand dans un environnement direct en influençant les individus dans leurs pratiques de mobilités, leurs activités consommatoires ou sociales. Ce cadre élargi limite la capacité à s'abstraire, à s'éloigner de l'activité qui marque la journée et le lieu, tout autant qu'il exerce en réaction une force de répulsion des activités et des liens sociaux privés. L'emprise exercée par le travail sur son environnement direct entraîne une répulsion à effectuer toute autre activité qui ne lui est pas liée.

\subsection{Un environnement de travail entre attraction et répulsion}

Le travail reste proche tant sur un plan spatial (impossibilité physique de trop s'éloigner) que temporel (la pause reste un moment borné de part et d'autre par les horaires du travail). Néanmoins cette proximité n'est pas subie par les travailleurs. Si la façon dont ils organisent leur pause témoigne de la force structurante du travail, cette dernière est liée au contexte temporellement limité qu'elle suppose. De fait, la force d'attraction du travail à son voisinage n'est que temporaire. Elle joue essentiellement pendant les pauses et en arrivant sur le site. Par contre, en dehors des horaires dits de travail, La Défense a un effet répulsif sur les personnes interrogées. L'activité professionnelle colore tellement le lieu que les individus souhaitent s'en soustraire au plus vite, s'en éloigner pour ne pas y revenir, dès que celle-ci est terminée. Le cadre du travail est tellement puissant qu'il annihile alors les autres cadres potentiels pouvant s'y exprimer, comme le cadre domestique ou le cadre des loisirs. Cette réalité a pour témoin l'attitude des personnes qui utilisaient le site avant d'y travailler :

Lorsque je ne travaillais pas à La Défense, il m'arrivait de venir sur le site pour faire les boutiques. Mais depuis que j'y travaille, je ne viens plus sur mon temps libre, ça me rappelle trop le travail.

(Femme, 27 ans, vendeuse de prêt-à-porter dans le centre commercial) 
Avant de travailler ici, il m'arrivait fréquemment de venir au ciné ou au Mac Do. Depuis je ne le fais plus car je déteste La Défense, je fuis le monde et le béton.

(Femme, 33 ans, employée dans une mutuelle)

Les activités réalisées sur le site après le travail s'avèrent rares alors même que le travail n'est plus censé retenir et normer les comportements des individus. Une fois la journée terminée, les personnes interrogées quittent La Défense pour rentrer chez elles ou joindre d'autres lieux. Celles qui utilisent l'offre du site déclarent des activités essentiellement domestiques, ne relevant pas des loisirs. Ainsi le soir est certes l'occasion d'aller boire un verre entre collègues, d'aller au cinéma, voire de faire des rencontres galantes. Mais de telles activités n'ont jamais lieu à La Défense. Elles sont effectuées à Paris, directement accessibles en transports en commun. Pour les autres activités, elles restent principalement «utilitaires »: La Poste, la banque, le dentiste, etc. Solliciter ces services sur son lieu de travail est opéré à défaut, par manque d'équipements autour du domicile ou par les horaires inadéquats de ces derniers.

Je vais à la FNAC mais c'est relativement rare. C'est surtout parce que la FNAC à côté de chez moi est fermée le soir quand je rentre.

(Homme, 44 ans, chef produit)

Je fais un tour dans les boutiques, surtout celles qu'il n'y a pas à côté de chez moi.

(Femme, 36 ans, chargée de clientèle dans les assurances)

II est bien difficile de s'abstraire de l'environnement de travail ou de lui appliquer un autre cadre qui ne lui est pas directement lié. D'abord, si l'on considère les activités domestiques observées comme des activités relevant d'un travail non rémunéré (Barrère-Maurisson et al., 200I), alors se produit une continuité thématique de la journée. Ensuite, les activités renvoyant au cadre des loisirs ou des relations amicales ne s'expriment guère à La Défense. Lorsqu'elles existent, elles s'affranchissent du lieu en le quittant physiquement (pour Paris ou la banlieue). Enfin, même lorsque des initiatives sont prises pour transformer La Défense (festival de jazz ou marché de Noël), faire rester les salariés et modaliser le cadre primaire du travail, les travailleurs ne s'y rendent guère :

J'avais vu un concert sur l'officiel qui avait lieu ici un week-end. Mais je n'y suis pas allé par choix. Retourner sur son lieu de travail, ce n'est pas agréable.

(Homme, 34 ans, chef de projet informatique)
Pour les responsables du site, l'implantation d'événements à La Défense a pour objectif d'attirer des visiteurs et de retenir les travailleurs afin d'animer l'endroit en dehors des horaires de bureau. Ces tentatives de festivalisation peuvent être apparentées à une modalisation du cadre de l'expérience (Goffman, 1991), c'est-à-dire « un ensemble de conventions par lesquelles une activité pourvue d'un sens dans l'application d'un cadre primaire, se transforme en une autre activité qui prend la première pour modèle mais que les participants considèrent comme différente ॥ (Goffman, 1991, p.52). La notion de cadre de l'expérience empruntée à Goffman et utilisée dans sa dimension spatiale s'articule avec celle de modalisation. La transformation du cadre spatial du travail aurait un effet sur les activités périphériques qui l'entourent en les transportant, temporairement et symboliquement, hors de leur cadre primaire. Les tentatives faites par l'EPAD (rebaptisé Defacto depuis le ${ }^{\text {er }}$ janvier 20l0) d'animer La Défense pour en faire un espace attractif sur un mode ludique peuvent-elles vraiment modifier les pratiques situées et rattachées au lieu de travail?

Le cas de La Défense est particulier mais interroge la capacité de l'environnement de travail à être transformé et à changer les routines qui lui sont rattachées. L'exercice semble finalement assez difficile à La Défense, qui reste constamment liée au cadre du travail. Toutefois, étudier la force du travail à sa proximité a été opéré dans un lieu qui lui est fortement dédié. Cette force serait-elle identique ou moindre dans un environnement différent, plus ouvert et moins rattaché, à première vue, au monde professionnel? En considérant le centre de Paris comme un autre terrain possible et Paris-Plages comme une forme de modalisation de ce cadre primaire, nous cherchons à tester l'élasticité du lien qui relie le travailleur à son adresse professionnelle. L'analyse des pratiques des travailleurs dans un cadre modalisé permet alors d'étudier la force de l'emprise du travail sur les routines, malgré la ludicisation des environnements de travail.

\section{LA DOMINATION DU TRAVAIL SUR LES TENTATIVES DE MODALISATION DE SON ENVIRONNEMENT : PARIS-PLAGES OU LA PRODUCTION D'UNE ROUTINE ESTIVALE}

Le travail qui influence et organise des routines spatiales et temporelles à proximité de l'adresse professionnelle est un cadre à l'action, à la fois social, temporel et physique. II marque le lieu et des activités de son sceau dont il est difficile de se défaire. 
L'aménagement de l'environnement de travail peut donc renforcer ce lien qui attache le lieu au travailleur, comme c'est le cas à La Défense où tout est organisé pour ce dernier. Ainsi, l'agent de service interrogé déplore que le béton, la difficulté à flâner et le manque de verdure du site ne lui permettent pas de se distancier psychologiquement de son activité professionnelle. Mais l'aménagement festif, ludique, récréatif de ce même environnement pourrait être à même de produire un cadre nouveau permettant une prise de distance avec le lieu de l'activité. L'événement ParisPlages, aménagement éphémère en centre-ville sur le mode balnéaire, est-il à même de contrer l'emprise du travail sur les pratiques spatiales à proximité? La transformation physique, symbolique et temporaire de l'espace par Paris-Plages permet-il de rompre avec le quotidien professionnel grâce à l'insertion d'un cadre ludique au sein du cadre professionnel? L'analyse des pratiques des travailleurs de cet événement montre finalement la capacité du travail à absorber la portée dépaysante de la transformation festive. Les routines du travail résistent aux tentatives de désorganisation.

\section{I La dimension répulsive de l'environnement de travail malgré Paris-Plages}

Comme pour La Défense, l'environnement autour du lieu de travail reste répulsif en dehors des périodes d'activité malgré la présence de Paris-Plages. D'abord, seulement $8 \%$ des travailleurs qui visitent l'opération déclarent le faire durant le week-end (WE), $47 \%$ d'entre eux privilégiant les visites en semaine. À l'inverse, les visiteurs qui ne sont pas au travail (RTT, congés annuels, inactifs, etc.) sont $18 \%$ à déclarer préférer venir le WE tandis que $27 \%$ visitent le site en semaine. Ensuite, le choix temporel des travailleurs est plus tranché, voire plus contraint : $54 \%$ des personnes effectuant leur visite durant leur temps libéré avouent ne pas avoir de préférence entre le WE et la semaine, ce n'est le cas que pour $44 \%$ des travailleurs. Ils expriment ici leur réticence à fréquenter l'environnement rattaché à leur adresse profes-sionnelle en dehors des jours de travail. Enfin, $75 \%$ des travailleurs interrogés à Paris-Plages sont célibataires et n'ont pas d'enfant (66\%), ce qui les oppose à la plupart des autres visiteurs, mariés et parents, qui pratiquent le site soit en famille, soit entre conjoints, en fin ou en milieu de semaine. Donc au-delà du caractère répulsif du lieu de travail, les travailleurs n'ont pas d'incitation relationnelle à y retourner dans le cadre événementiel. Leur visite correspond soit à une pause au sein de la journée de travail, soit à un détour entre le travail et le domicile à la fin de la journée (cf. tableau I).

Ces modalités de visite dépendent de la distance de l'événement au lieu de travail, ce qui marque l'existence d'une emprise du travail sur un périmètre spatial qui détermine les pratiques individuelles. Plus la distance entre Paris-Plages et le travail est faible, plus les travailleurs y viennent en pause et n'y retournent pas lorsqu'ils ont terminé leur journée. La fin de la journée de travail signifie alors la fin de la présence dans le quartier, malgré le caractère ludique de l'événement qui en transforme l'ergonomie et l'ambiance. Le périmètre «ludicisé » perd de son attractivité car il est dominé par le caractère répulsif du cadre spatial associé au travail. À l'inverse, lorsque

Tableau I

Localisation du lieu de travail et moment de la visite de Paris-Plages (en \%, tous sites confondus, échantillon des travailleurs)*

Type de visite

\begin{tabular}{|c|c|c|c|c|c|}
\hline & & $\begin{array}{c}\text { Fin de la période de } \\
\text { travail }\end{array}$ & $\begin{array}{c}\text { Pause dans le } \\
\text { travail }\end{array}$ & Total & Effectifs \\
\hline Lieu de & $\begin{array}{l}\text { Arrondissements accueillant } \\
\text { Paris-Plages }\end{array}$ & 23 & 77 & 100 & 72 \\
\hline travail & Autres arrondissements & 54 & 46 & 100 & 75 \\
\hline & Hors Paris & 73 & 27 & 100 & 41 \\
\hline & Total & 46 & 54 & 100 & 188 \\
\hline
\end{tabular}


l'opération se localise hors de l'aire d'influence du travail, les travailleurs l'utilisent comme un lieu de sortie à la fin de leur journée. L'élasticité de l'emprise psychologique du travail dans l'espace est inversement proportionnelle à la distance de l'adresse professionnelle. Le cadre du travail possède des gradients exprimés en termes d'influence sur les pratiques socio-spatiales. Ces gradients décroissent à mesure que l'individu s'éloigne de son adresse professionnelle. Ils démontrent qu'il est difficile de modaliser le cadre du travail à son voisinage. Cependant, les possibilités d'éloignement du travail dépendent aussi des offres de mobilités, plus ou moins rapides, qui permettent de se mettre à distance physique de l'adresse professionnelle. On peut faire l'hypothèse que les possibilités de déplacement, alliées aux contraintes de l'agenda professionnel, distordent le périmètre où se déploie l'emprise du travail et lui donnent une forme qui s'étend le long des lignes de transport.

L'accessibilité temporelle à Paris-Plages depuis le lieu de travail influence davantage les personnes en situation d'activité à s'y rendre que cette même proximité au domicile n'influence celles en situation de congé ou d'inactivité. Le temps de déplacement pour se rendre à Paris-Plages est d'autant plus explicatif de la présence des travailleurs qu'il est faible (cf. tableau 2). Un temps de parcours faible permet d'articuler les contraintes inhérentes à la journée de travail avec le besoin de s'échapper de son cadre spatial. Cela n'est pas le cas pour les autres profils, qui assument un temps de déplacement plus long. L'addition à l'effet de proximité spatiale d'un effet de proximité temporelle, illustre un peu plus la force d'attraction du travail sur son environnement et, par conséquent, sur les pratiques de Paris-Plages. Plus le temps de trajet est long, plus le temps de visite des travailleurs prend de l'importance : $56 \%$ des travailleurs à moins de dix minutes de l'événement restent moins d'une heure contre $42 \%$ qui se trouvent entre dix et 30 minutes de trajet et $27 \%$ de ceux à plus de 30 minutes. À l'inverse, le temps de présence augmente avec le temps de trajets: à moins de dix minutes de trajet, $45 \%$ des travailleurs restent plus d'une heure; entre dix et 30 minutes, ils sont $57 \%$ et à plus de 30 minutes, $63 \%$ d'entre eux restent plus d'une heure.

La différenciation des pratiques qui se fait sentir lorsque le temps de trajet dépasse la demi-heure s'explique notamment par le moment de la visite qui correspond à la fin des contraintes temporelles du travail. Plus le temps de déplacement pour se rendre à Paris-Plages est long, plus il grignote potentiellement le temps libre au cœur de la journée de travail, et plus les travailleurs reportent leur visite en fin de journée (cf. tableau 3).
Lorsque la durée de déplacement est inférieure à dix minutes, la grande majorité des travailleurs utilisent l'événement pour prendre leur pause. Au-delà de ça, ils sont plus nombreux à reporter leur visite en fin de journée. Ne pouvant que difficilement articuler un temps de déplacement long avec un temps de pause généralement court, les travailleurs cherchent à maximiser l'utilité de ce dernier en rompant avec le cadre spatial du travail par l'insertion dans un cadre ludique. L'élasticité que le travailleur entretient avec l'espace-temps professionnel n'est cependant pas la même pour toutes les catégories socioprofessionnelles : $60 \%$ des artisans, commerçants et chefs d'entreprise restent moins de deux heures sur le site, $87 \%$ sont en situation de pause et $73 \%$ d'entre eux déclarent ne pas avoir programmé leur visite. En revanche, $41 \%$ des employés restent moins de deux heures, $50 \%$ sont en pause et $72 \%$ programment leur visite. Ces différences s'expliquent par les marges de liberté dont les travailleurs disposent dans l'organisation de leur journée de travail. Plus ou moins subies, ces marges illustrent l'élasticité variable qu'exerce la force du travail sur les pratiques spatiales. Globalement, la visite est plus programmée chez les non travailleurs que chez les travailleurs. Pour ces derniers, la pratique de l'événement est une opportunité à saisir, de manière plus ou moins contrainte par la nature de l'activité, dans l'optique d'un éloignement avec l'emprise du quotidien professionnel.

Le difficile arbitrage entre la volonté de sortir de l'environnement de travail, le temps réduit de la pause, et le temps de déplacement prêt à être assumé pour se rendre à Paris-Plages traduit le poids du travail sur les pratiques spatiales et la faible élasticité temporelle qu'il autorise. Désormais, travail et plaisir, productivité et loisirs se combinent de manière non exclusive, se décloisonnent et s'articulent (Gravari-Barbas, 200I). Mais le travail continue d'orienter les pratiques spatiales par une importante force de rappel malgré cette «accumulation de symboles et d'objets qui constituent un jeu de mécano qui va permettre aux gens de croire à l'espace. [...] On thématise un espace pour que les gens y croient en très peu de temps » (Choblet, voir la note 2). La coloration par le travail de son environnement direct, qui lui donne son caractère répulsif en dehors des périodes d'activités, domine les tentatives de coloration ludique du même espace. 
EUE • Des environnements urbains sous influence $\bullet$ c-30

Tableau 2

Type de journée et temps de déplacement pour se rendre à Paris-Plages

(en \%, tous sites confondus)

Temps de déplacement depuis le lieu de travail

\begin{tabular}{|c|c|c|c|c|c|c|c|}
\hline & & $\begin{array}{l}\text { Moins de dix } \\
\text { minutes }\end{array}$ & $\begin{array}{l}\text { Entre dix et } \\
30 \text { minutes }\end{array}$ & $\begin{array}{c}\text { Entre } 30 \\
\text { minutes et une } \\
\text { heure }\end{array}$ & $\begin{array}{l}\text { Plus } \\
\text { d'une } \\
\text { heure }\end{array}$ & Total & Effectifs \\
\hline & $\begin{array}{l}\text { Inactif ou actif non } \\
\text { occupé }\end{array}$ & 14 & 43 & 32 & 10 & 100 & 166 \\
\hline \multirow[t]{3}{*}{ Profils } & $\begin{array}{l}\text { Journée non } \\
\text { travaillée }\end{array}$ & 20 & 49 & 24 & 8 & 100 & 1157 \\
\hline & Journée de travail & 29 & 49 & 19 & 3 & 100 & 187 \\
\hline & Total & 21 & 48 & 24 & 7 & 100 & 1510 \\
\hline
\end{tabular}

Tableau 3

Temps de déplacement et moment de la visite à Paris-Plages des travailleurs

(en \%, tous sites confondus, échantillon des travailleurs)

Type de visite

\begin{tabular}{|c|c|c|c|c|c|}
\hline \multirow[b]{2}{*}{ Temps de } & & Fin de journée de travail & Pause dans le travail & Total & Effectifs \\
\hline & Moins de dix minutes & 29 & 71 & 100 & 61 \\
\hline déplacement & Entre dix et 30 minutes & 55 & 45 & 100 & 88 \\
\hline depuis le lieu de & Plus de 30 minutes & 48 & 52 & 100 & 39 \\
\hline travail & $\overline{\text { Total }}$ & 46 & 54 & 100 & 188 \\
\hline
\end{tabular}


Tableau 4

Type de journée et fréquence de la visite estivale

(en \%, site des Voies sur berges)

Nombre de visites

\begin{tabular}{|c|c|c|c|c|c|c|c|}
\hline & & $\begin{array}{l}\text { Une ou deux } \\
\text { fois }\end{array}$ & $\begin{array}{l}\text { Trois ou } \\
\text { quatre fois }\end{array}$ & $\begin{array}{c}\text { Plus de } \\
\text { quatre fois }\end{array}$ & $\begin{array}{l}\text { Ne sait } \\
\text { pas }\end{array}$ & Total & Effectifs \\
\hline \multirow{4}{*}{ Profils } & Journée non travaillée & 27 & 45 & 25 & 3 & 100 & 431 \\
\hline & Journée de travail & 10 & 48 & 39 & 4 & 100 & 84 \\
\hline & Inactif ou actif non occupé & 20 & 51 & 29 & n.s. & 100 & 95 \\
\hline & Total & 24 & 47 & 27 & 2 & 100 & 610 \\
\hline
\end{tabular}

Tableau 5

Distance du lieu de travail de Paris-Plages et fréquence de la visite estivale (en \%, tous sites confondus, échantillon des travailleurs)

Nombre de visites

\begin{tabular}{|c|c|c|c|c|c|}
\hline & & Mons de trois visites & Plus de trois visites & Total & Effectifs \\
\hline & $\begin{array}{l}\text { Arrondissements accueillant Paris- } \\
\text { Plages }\end{array}$ & 39 & 61 & 100 & 72 \\
\hline Lieu de & Autres arrondissements & 53 & 47 & 100 & 75 \\
\hline \multirow[t]{2}{*}{ travail } & Hors Paris & 77 & 23 & 100 & 41 \\
\hline & Total & 52 & 48 & 100 & 188 \\
\hline
\end{tabular}

Tableau 6

Type de journée et temps de présence à Paris-Plages

(en \%, tous sites confondus)

Temps de visite

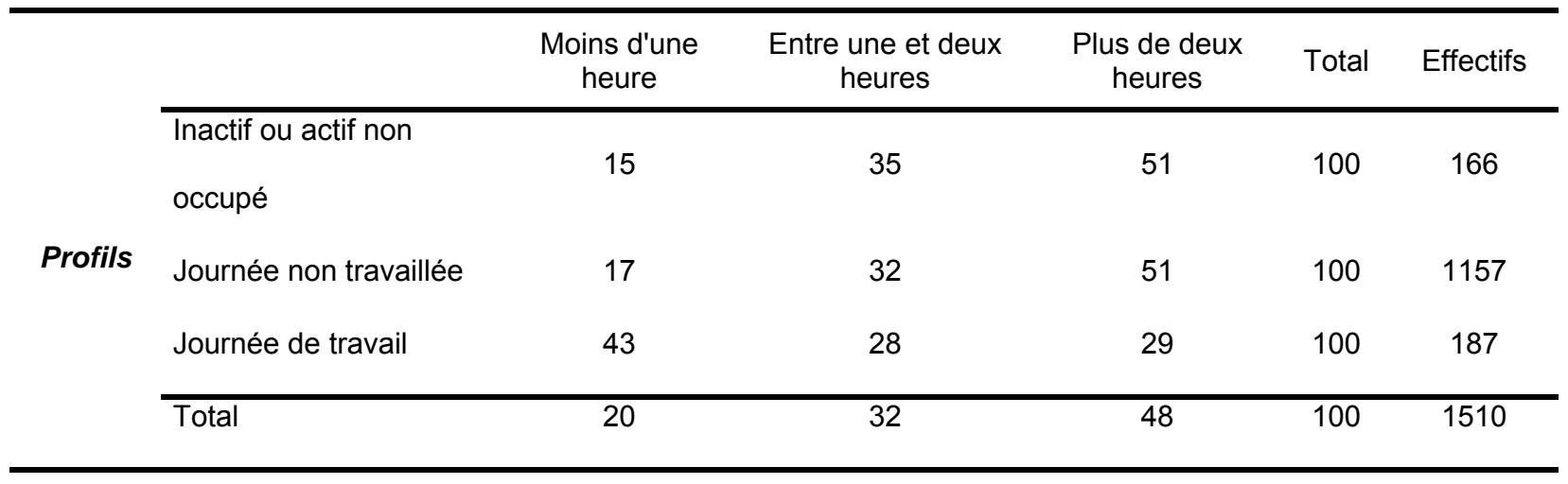


EUE • Des environnements urbains sous influence $\bullet$ c-32

Tableau 7

Mode de fréquentation de Paris-Plages par les travailleurs et sociabilité (en\%, tous sites confondus)

Interactions sociales

\begin{tabular}{|c|c|c|c|c|c|}
\hline & & $\begin{array}{c}\text { Aucune rencontre } \\
\text { ni échange }\end{array}$ & $\begin{array}{l}\text { Au moins une } \\
\text { rencontre ou un } \\
\text { échange }\end{array}$ & Total & Effectifs \\
\hline Types & de Pratique en groupe & 56 & 44 & 100 & 109 \\
\hline \multirow[t]{2}{*}{ pratique } & Pratique en solitaire & 39 & 61 & 100 & 74 \\
\hline & Total & 49 & 51 & 100 & 183 \\
\hline
\end{tabular}

Tableau 8

Proximité de Paris-Plages au travail et perception de la foule (en \%, tous sites confondus, échantillon des travailleurs)

Perception de la fréquentation

\begin{tabular}{|c|c|c|c|c|c|c|}
\hline \multirow{5}{*}{$\begin{array}{l}\text { Lieu de } \\
\text { travail }\end{array}$} & & $\begin{array}{l}\text { Il y a juste le } \\
\text { monde qu'il faut }\end{array}$ & $\begin{array}{c}\text { Il n'y a pas } \\
\text { assez de } \\
\text { monde }\end{array}$ & $\begin{array}{l}\text { Il y a trop de } \\
\text { monde }\end{array}$ & Total & Effectifs \\
\hline & $\begin{array}{l}\text { Arrondissements accueillant Paris- } \\
\text { Plages }\end{array}$ & 63 & 26 & 11 & 100 & 64 \\
\hline & Dans d'autres arrondissements & 78 & 12 & 10 & 100 & 69 \\
\hline & Hors Paris & 82 & 7 & 11 & 100 & 32 \\
\hline & Total & 73 & 16 & 11 & 100 & 165 \\
\hline
\end{tabular}




\subsection{L'absorption de Paris-Plages par la routine liée aux contraintes du travail}

Parce que Paris-Plages a intégré les routines estivales des travailleurs, sa capacité à transformer le cadre urbain associé au travail s'affaiblit d'année en année. Le « city marketing » (Ingallina et Park, 2005) qui ludicise les centres villes rapproche physiquement le travail des activités de loisirs. II s'appuie sur les actifs occupés qui intègrent les événements dans leurs pratiques quotidiennes. L'extension de Paris-Plages à la ZAC du Bassin de la Villette (59500 $\mathrm{m}^{2}$ de bureaux) et à la ZAC de Paris Rive Gauche $\left(448300 \mathrm{~m}^{2}\right.$ de bureaux) rapproche le loisir des lieux de l'activité économique. L'équipement en Wifi et le Café-WifiJournaux offrent la possibilité de travailler sur place et de rester connecté au travail. La multiplication des points de restauration rapide, les buvettes avec terrasses et les activités peu chronophages (promenade, pétanque, lecture) offrent des lieux de pause inédits qui peuvent s'intégrer aux routines spatiales estivales des actifs.

Plus qu'une recombinaison entre travail et loisirs, la routinisation de l'événement illustre son absorption par le travail et cela à plusieurs échelles temporelles. À l'échelle pluriannuelle, les travailleurs intègrent l'événement dans leurs pratiques spatiales estivales autour de leur lieu d'activité. La proportion des travailleurs qui ont l'habitude de fréquenter l'opération chaque année, depuis 2002, est inférieure à celle des inactifs mais légèrement supérieure aux personnes en situation de congé. Et si $68 \%$ des travailleurs ont déjà fréquenté l'opération au moins une fois lors des précédentes éditions, ils sont une grande majorité (73\%) à affirmer qu'ils reviendront à l'édition suivante. Cette routinisation de la visite annuelle dans le cadre du travail - et rarement en dehors, se combine avec une routinisation à l'échelle de l'événement étudié en 2007.

Les travailleurs font plus de visites à Paris-Plages que les personnes en congé ou inactives (cf. tableau 4). Ils constituent une population assidue, routinière, connaissant bien les lieux et leurs aménités. Un travailleur sur deux s'est rendu plus de quatre fois à Paris-Plages 2007 sur le site le plus ancien et connu (Voies sur Berges). L'événement influe sur l'organisation estivale des activités effectuées à la fin de la journée de travail, mais plus certainement sur l'organisation des pauses quotidiennes. Si $38 \%$ des travailleurs qui viennent plus de quatre fois à ParisPlages ont fini leur journée, $62 \%$ sont en situation de pause. Cette multiplication des pauses est rendue possible par la proximité de l'événement au lieu de travail (cf. tableau 5). Elle illustre la faible élasticité du rapport spatiotemporel qu'entretient le travailleur avec son lieu d'activité.

Les travailleurs sont également les plus décidés à retourner à Paris-Plages puisque $83 \%$ d'entre eux ont l'intention de revenir d'ici la fin de l'été 2007 contre $73 \%$ de personnes en situation de congés et $76 \%$ des inactifs. La routinisation de la pratique de l'événement affaiblit son pouvoir d'éloignement d'avec le cadre social et spatial du travail. La multiplication des visites s'explique aussi par les activités réalisées qui s'articulent avec les besoins du travail. Les travailleurs se singularisent par une utilisation fonctionnelle de l'événement puisque $39 \%$ d'entre eux ont profité de l'offre de restauration contre $27 \%$ des non travailleurs. De même, $15 \%$ des répondants au travail ont cité cette offre comme élément explicatif de leur visite contre $3 \%$ des usagers en vacances et $1 \%$ des inactifs. Restauration, promenade et utilisation des transats pendant la pause constituent $66 \%$ des activités effectuées par les travailleurs. Elles peuvent être facilement interrompues pour retourner au travail à l'inverse des ateliers créatifs, des sports en équipes ou des concerts. Ces activités donnent une identité à la visite mais permettent aux travailleurs de regagner rapidement leur activité, voire de rentrer vite chez eux après leur journée parce qu'ils ne souhaitent pas s'éterniser dans leur environnement de travail. Ils restent ainsi bien moins longtemps à Paris-Plages que les autres profils de visiteurs (cf. tableau 6).

Pour une pause pour manger un morceau dans le cadre de la journée de travail ou une rapide promenade avant de rentrer au domicile, les travailleurs ont adopté Paris-Plages. Ces fonctions rappellent celles du café dans une certaine tradition ouvrière, lieu de transit entre l'usine et le foyer et lieu de pause pour se retrouver (Pillon, 1997). Au final, la pratique de Paris-Plages ne s'extrait pas de l'emprise du travail. Elle s'intègre dans l'environnement spatial du lieu d'activité et l'organisation temporelle de la journée. D’année en année, l'événement revêt un caractère fonctionnel qui lui fait perdre en partie sa capacité à évoquer autre chose que le quotidien. Une routine annuelle est instaurée. La coupure avec l'environnement de travail se joue ailleurs.

\subsection{Se mélanger à la foule événementielle : une autre manière de prendre de la distance au travail}

La visite à Paris-Plages peut être interprétée comme une pratique de délocalisation de la pause et de mise à distance de la journée de travail à travers 
l'endossement d'un nouveau rôle permettant une reprise de soi-même (Hatzfel, 2002). Mais qu'est ce qui fait que les travailleurs se rendent là et pas dans des lieux calmes où l'on peut s'extraire de la foule comme les parcs et jardins (Sansot, 2003) ou les terrasses des petites rues piétonnes de certains quartiers peu fréquentés?

Les attraits de Paris-Plages exprimés par les travailleurs diffèrent sensiblement de ceux déclarés par les autres profils : $34 \%$ des travailleurs estiment que l'opération permet de «briser le quotidien » contre $26 \%$ des personnes hors travail ; $15 \%$ insistent sur l'intérêt de la forte fréquentation et $38 \%$ sur l'animation que l'événement apporte à la ville contre respectivement $12 \%$ et $35 \%$ pour les non travailleurs. La rupture avec l'environnement de travail connu et pratiqué quotidiennement participe de l'intérêt de l'opération pour les travailleurs. Mais, ils se sentent logiquement moins en vacances à Paris-Plages que les visiteurs en congé ou inactifs. Si la fréquentation est un autre élément d'intérêt pour les travailleurs, ils cherchent moins à faire des rencontres qu'à vivre leur été en ville de manière collective. Ils sont sensibles à la publicité estivale de Paris-Plages. Pour eux, la convivialité $(56 \%)$ et la saisonnalité (56\%) qualifient le mieux l'ambiance de l'événement, suivies par son aspect reposant et calme (52\%). À l'inverse de La Défense où les pratiques socialisatrices sont rares et où l'espace se vide le soir, Paris-Plages installe une «convivialité estivale» et une fréquentation hors norme à proximité du travail. Le rassemblement festif influence la localisation de la pause. Pour les $33 \%$ de travailleurs venus «entre amis » à Paris-Plages, on peut penser que "Le moment du repas apparait comme un moment de grande dispersion de l'équipe. Les groupes qui s'établissent alors se fondent sur d'autres liens que ceux du travail. » (Hatzfel, 2002).

Les liens sociaux s'expriment sur un registre alternatif au travail et se renforcent par le contact avec une foule d'anonymes (cf. tableau 8): seuls $44 \%$ d'entre eux avouent avoir discuté ou fait une rencontre sur le site. À l'inverse, pour $40 \%$ des travailleurs seuls, la convivialité et la foule semblent permettre de rompre avec le groupe du travail : 61 \% d'entre eux ont rencontré ou eu un échange verbal avec quelqu'un durant leur visite. La foule procure un anonymat permettant d'endosser un autre rôle que celui joué sur le lieu d'activité. Elle offre la possibilité de rencontrer ou, du moins, d'observer des individus n'appartenant pas à la sphère de travail et dans un cadre dépaysant.
À la transformation physique du cadre du travail, la fréquentation hors norme de Paris-Plages à sa proximité produit une transformation de l'ambiance sociale de l'espace rattaché au lieu d'activité. II y a donc une triple rupture: celle d'avec les relations sociales au sein de l'espace de travail, celle avec les modalités de l'interaction au quotidien dans les espaces publics faites de distance polie et d'évitement, celle enfin avec la faible fréquentation de Paris pendant l'été.

Plus les travailleurs sont localisés près de ParisPlages, plus ils souhaitent que l'événement attire du monde et augmente l'animation de leur environnement direct (cf. tableau 8). Ainsi, $50 \%$ des travailleurs ont fait une rencontre sur le site, dont $45 \%$ avec une personne qu'ils ne connaissaient pas. Et une faible fréquentation devient un élément peu attractif pour eux. Paris-Plages ne déstructure pas les routines du travail mais la fréquentation que l'opération engendre dans l'environnement du travail offre une ambiance propice à la délocalisation des relations de travail, voire à leur redéfinition.

La force de rappel du lieu de travail influence les pratiques spatiotemporelles des travailleurs en visite à Paris-Plages. Le cadre du travail déborde de l'adresse professionnelle pour se répandre dans un périmètre variable autour du lieu d'activité, contraignant les pratiques et engendrant des routines qui s'intègrent au travail. Cependant, la densité de fréquentation que génère l'événement change l'ambiance de l'environnement de travail et permet d'opérer une forme de distanciation d'avec le quotidien professionnel. La coprésence entraînée par la transformation ludique de la ville permet d'expérimenter une altérité urbaine autre que celle liée au travail et au cadre de la ville quotidienne dans lequel il s'ancre à l'année. « Ce qui se cherche ici, et qui se trouve, c'est un tropisme des pratiques urbaines, une propension à 'fréquenter les fréquentations' » (Bordreuil, 2004). Paris-Plages est un rendez-vous saisonnier, aujourd'hui routinier, qui ouvre aux travailleurs la possibilité de redéfinir les modalités des relations de travail à proximité du travail, d'expérimenter des espaces publics inhabituellement usités et de changer de l'ambiance sociale de l'environnement de travail connu et routinier. 


\section{CONCLUSION}

La modernité urbaine caractérisée par une grande liberté individuelle dans l'organisation spatiotemporelle des séquences d'activités et par le brouillage des frontières entre des types d'activités entraînerait l'affaiblissement des pratiques routinières, spatialement structurées autour de quelques lieux centraux. Ce constat s'appliquerait notamment à la sphère du travail, plus flexible et plus délocalisée. Or on perçoit à travers ces deux études la persistance du poids du travail, de son organisation temporelle et de sa localisation dans la structuration des modes de vie et dans la répétition des pratiques spatiales. La force à la fois attractive et répulsive du lieu de travail pèse sur les déplacements dans son environnement sous influence et au-delà. Des routines organisationnelles quotidiennes continuent de structurer les usages de l'espace associé au lieu de travail. Elles répondent à des impératifs d'ordre utilitaire ou domestique. Si l'élasticité qui relie l'individu à son lieu de travail paraît de plus en plus grande dans les représentations de la modernité actuelle, elle ne rompt pas totalement et conserve une portée analytique pertinente. Le lieu de travail continue de colorer spatialement, temporellement et psychologiquement son environnement direct. Cette élasticité est si forte qu'elle ne rompt pas face aux transformations événementielles de l'environnement de travail qui pourraient pourtant influer sur l'organisation des pratiques qui lui sont rattachées.

Pour autant, ces deux enquêtes nous permettent essentiellement de saisir les comportements d'individus qui sortent de leurs lieux de travail, de leurs bureaux, pour effectuer leur pause. En les interrogeant dans l'espace public, nous ne pouvions saisir les pratiques et motivations de ceux qui restent au travail ou fréquentent des lieux autres que ceux enquêtés ici. La première catégorie de personnes est l'archétype de ceux sur lesquels la force du travail est la plus forte, puisqu'ils ne s'en éloignent pas. La seconde catégorie ne fait pas toujours les mêmes types de pauses: ils peuvent articuler d'une fois sur l'autre plusieurs activités, plusieurs lieux différents. Ainsi disséminés, il est difficile de les appréhender comme une catégorie univoque.

C'est notamment pour conserver ces catégories à proximité du travail en dehors des horaires de bureau et amener les autres à revenir en familles les jours de congés que l'établissement public en charge de la gestion et de l'animation de La Défense (ex-EPAD) s'est engagé dans la production d'ambiances festives permettant une mise à distance de l'environnement de travail. À l'image de Paris-Plages et de ses activités gratuites en centre ville, des installations temporaires inédites ont été commandées au scénographe de ParisPlages par Defacto. Outre la multiplication d'événements sur la dalle de La Défense (I8 en 2008) qui doivent stimuler de nouvelles formes d'investissement du site, une Biennale Internationale de Mobilier Urbain précédée pendant un an d'installations temporaires a pour objectif de "recréer une 'vraie' ville avec de 'vraies' gens au pied des tours ${ }^{3} »$. La présence de ces «vraies gens», qui semblent s'apparenter aux non travailleurs doit être stimulée par un rehaussement de la qualité de l'espace public de La Défense, tout comme Paris-Plages devait produire « un espace de qualité, un espace vivant et fréquenté, animé, transformant une autoroute urbaine en lieu partagé ${ }^{4}$. L'objectif de stimuler une forme de mixité des utilisateurs des lieux semble prendre le pas sur la dimension matérielle de l'aménagement dans l'explication de la possibilité d'un décadrage d'avec le quotidien urbain en général, et du cadre de travail en particulier. Plus que les transformations événementielles de l'environnement de travail, ce sont avant tout les modes d'investissement collectif de l'espace qu'elles induisent qui permettent une prise de distance avec l'activité quotidienne et ses routines solitaires.

Néanmoins, la possibilité de prendre du recul dans la journée de travail, voire de brouiller les habitudes par l'immersion dans un être ensemble inhabituel, est subordonnée aux routines liées au travail. La double dimension attractive/répulsive de l'adresse professionnelle et la coloration qu'elle engendre sur son environnement direct dominent les comportements hors travail. La pratique de l'événement devient une routine estivale et annuelle. Le quotidien du travail absorbe l'exception de l'événement en l'intégrant à ses logiques organisationnelles. Ludiciser le centre ville ou le quartier d'affaire ne permet pas de faire disparaitre l'influence du travail, ni dans la signification, ni dans les pratiques associées à son environnement direct. Ce qui prouve que l'activité professionnelle continue d'avoir une emprise forte sur les pratiques spatiales, temporelles et sociales à sa proximité immédiate.

\footnotetext{
${ }^{3} \mathrm{H}$. Van Melle, directeur de l'agence Division Créative en charge par l'EPAD de l'animation de La Défense, « La Défense contreattaque », I4/04/05, www.stratégie.fr

${ }^{4}$ B. Gouyette, Secrétariat générale mairie de Paris, responsable de la mission qualité espace public et Pilote de l'opération Paris Plage en 2002 et 2003. Entretien réalisé en septembre 2007.
} 


\section{BIBLIOGRAPHIE}

Aguilera, A., M.-H. Massot et L. ProulhaC (20I0). «Travailler et se déplacer au quotidien dans une métropole. Contraintes, ressources et arbitrages des actifs franciliens », Sociétés Contemporaines, $n^{\circ} 80$, p.29-45.

AsChER, F. (2004). « Le futur au quotidien. De la fin des routines à l'individualisation des espaces-temps quotidiens ", in AUBERT, N. (dir.), L'Individu Hypermoderne, Toulouse, Eres, p. 273-290.

Barrere-Maurisson, M.-A., S. Rivier et C. MinNi (200I). « Le partage du temps pour les hommes et les femmes ou comment conjuguer travail rémunéré, travail non rémunéré et non travail $"$, Premières Informations, Premières Synthèses, $n^{\circ}$ II-I, DARES, $8 \mathrm{p}$.

BaUmaN, Z. (2007). Liquid Time: Living in an Age of Uncertainty, Cambridge, Polity Press, 128 p.

BORDREUIL, J.-S. (2004). « Faire la ville sur les flux? Faire ville dans les flux. Entrer dans la compagnie des passants», actes du colloque Faire la ville avec les flux, Paris, Institut de la Ville en Mouvement, 29 et 30 mars, [En ligne] URL : www.ville-en-mouvement.com, site consulté le 04 mars $201 \mathrm{I}$

CAIRNCROSS, F. (200I). The Death of Distance, How the Communications Revolution Will Change Our Lives, Cambridge, Harvard Business School Press, 302 p.

Cette, G. (2004). « TIC portables et conciliation des temps sociaux $»$, Tempos, $n^{\circ} 3$, p. 38-42.

Crague, G. (2004). «Des lieux de travail de plus en plus variables et temporaires ॥, Économie et Statistiques, $\mathrm{n}^{\circ}$ 369-370, p. 191-212.

DE CONINCK, F. (20I0). "Pourquoi subir quand on peut choisir? », in M.-H. Massot (dir.), Mobilités \& modes de vie métropolitains. Les intelligences du quotidien, Paris, L'œil d'Or, p. 2II-2I7.

Delahaye, H. et C. Guillot, (2010). «Trois formes de routines au cœur de la (re)synchronisation des temps sociaux »), in M.-H. MASSOT (dir.), Mobilités \& modes de vie métropolitains. Les intelligences du quotidien, Paris, L'œil d'Or, p. 263-278.

GUÉRIN-PACE, P. (2003). « Vers une typologie des territoires urbains de proximité », L'Espace Géographique, $\mathrm{n}^{\circ} 4$, p.333-344.

Goffman, E. (199|). Les cadres de l'expérience, Paris, Les éditions de Minuits, $570 \mathrm{p}$.

GravarI-BARBAS, M. (200I). « Les nouveaux loisirs créent-ils un nouvel urbanisme », site du festival international de géographie de Saint-Dié-des-Vosges: http://fig-stdie.education.fr/actes/actes 200I/barbas/article.htm, site consulté le 04 mars $201 \mathrm{I}$.

Hatzfel, N. (2002). « La pause casse-croûte. Quand les chaînes s'arrêtent à Peugeot Sochaux ॥, Terrain : Travailler à l'usine, $n^{\circ} 39$, p. 33-48.
JAURÉGUIBERRY, F. (2003). « L'homme branché, mobile, pressé » in F. ASCHER et F. GODARD (dirs.), Modernité la nouvelle carte du temps, La Tour d'Aigues, Éditions de l'Aube/DATAR, p. I55-169.

JAVEAU, C. (2006). «Routines quotidiennes et moments fatidiques $»$, Les cahiers internationaux de la sociologie, $\mathrm{n}^{\circ}$ 221 , p. $227-238$.

LAïDI, Z. (2002). « Le temps de l'urgence » in J.-Y. BOULIN, P. DOMMERGUES et F. GODARD (dirs.), La nouvelle aire du temps, Paris, Éditions de l'Aube/DATAR, p. 5I-59.

LICOPPE, C. (2002). «Sociabilité et technologies de la communication. Deux modalités d'entretien des liens interpersonnels dans le contexte du déploiement des communications mobiles $\gg$, Réseaux, $\mathrm{n}^{\circ} 112-113, \mathrm{p}$. 172210 .

INGALLINA, P. et J. PARK (2005). « Les nouveaux enjeux de l'attractivité urbaine », Urbanisme, $\mathrm{n}^{\circ}$ 344, p. 64-68.

Pillon, T. (1997). "Individualisation du temps de travail et espaces publics », Annales de la Recherche Urbaine, $n^{\circ} 77$, p. 53-58.

SANSOT, P. (2003). Les jardins publics, Paris, Payot, 272 p.

URRY, J. (2005). Sociologie des mobilités. Une nouvelle frontière pour la sociologie? Paris, Armand Colin, 256 p.

VIARD, J. (2002). Le sacre du temps libre, la société des 35 heures, Paris, Éditions de l'Aube, 216 p. 